\title{
A New Integral Inequality and Delay-Decomposition with Uncertain Parameter Approach to the Stability Analysis of Time-Delay Systems
}

\author{
Haiyang Zhang, ${ }^{1,2}$ Lianglin Xiong, ${ }^{1,3}$ Qing Miao, ${ }^{1}$ Yanmeng Wang, ${ }^{1}$ and Chen Peng ${ }^{1}$ \\ ${ }^{1}$ School of Mathematics and Computer Science, Yunnan Minzu University, Kunming 650500, China \\ ${ }^{2}$ School of Science, Nanjing University of Science and Technology, Nanjing 210094, China \\ ${ }^{3}$ School of Mathematics and Statistics, Yunnan University, Kunming 650091, China \\ Correspondence should be addressed to Lianglin Xiong; lianglin_5318@126.com
}

Received 25 July 2015; Accepted 24 January 2016

Academic Editor: Everaldo M. Bonotto

Copyright ( 2016 Haiyang Zhang et al. This is an open access article distributed under the Creative Commons Attribution License, which permits unrestricted use, distribution, and reproduction in any medium, provided the original work is properly cited.

\begin{abstract}
This paper is concerned with the problem of delay-dependent stability of time-delay systems. Firstly, it introduces a new useful integral inequality which has been proved to be less conservative than the previous inequalities. Next, the inequality combines delay-decomposition approach with uncertain parameters applied to time-delay systems, based on the new Lyapunov-Krasovskii functionals and new stability criteria for system with time-delay have been derived and expressed in terms of LMIs. Finally, a numerical example is provided to show the effectiveness and the less conservative feature of the proposed method compared with some recent results.
\end{abstract}

\section{Introduction}

In recent years, the investigation about the stability of timedelay systems has already become a nuclear problem with the emergence of both suitable theoretical tools and more complex practical issues in the engineering field and information technology such as ecology, economics, or biology (see [1-6]). For the linear delay system, the stability conditions of systems are derived by using a lot of techniques. Among them, there are two most popular different approaches that allowed us to gain more efficient criteria. The first one is a classical methodology based on the survey of the roots of the related characteristic equation (see [7-11]); it is very effective in practice (see [12-14]) and perfect describer of the stability of systems. To some extent, however, this process exposes some limitations of itself that it cannot be spread straightway to other cases: the robust case and the system with time varying delay. Another one is the use of Lyapunov-Krasovskii functionals. As we all know, their general structures are simple, but the numeric is difficult to operate (see [15-19]). Furthermore, if some additional hypotheses are formulated on the Lyapunov functional, we can find that some conservative results are expressed in terms of LMIs.
Generally, we resolve these problems by either choosing extended state based on Lyapunov-Krasovskii functional (see [19-23]) and/or discretized Lyapunov functional (see [6-8]) and/or improving the existing integral inequality (see [21, $22,24-26]$ ). But, beyond that, another important technique reduces the conservatism, which is to bound some cross terms that have arisen when computing the derivative of Lyapunov functional. Looking through the literature for this purpose (see $[23,25,27,28]$ ), the similar characteristic of all these approaches is a resort to the slack variables (see $[21,22,24]$ ) and Jensen's inequality (see [29-32]).

Fortunately, to the best of the authors' knowledge, we proposed a new more accurate inequality, which is proved to be less conservative than the existing inequalities based on Jensen's theorem, and constructed a more simple LyapunovKrasovski functional by combining delay-decomposition approach with uncertain parameter in this paper. It is worth noting that this new functional depends not only on $x(t)$ and $x(t-h)$ but also on $x(t-\alpha h), \int_{t-\alpha h}^{t} x(s) d s$, and $\int_{t-h}^{t-\alpha h} x(s) d s$, such that we can get more better result. Then, the last signal is directly integrated into a new appropriate LyapunovKrasovskii functional to highlight the feature of the new 
inequality. Finally, new stability criteria for system with timedelay will be derived and will be expressed straightaway in terms of LMIs.

Notations. Throughout the paper, $R^{n}$ denotes the $n$ dimensional Euclidean space with vector norm $|\cdot|$, and $R^{n \times m}$ is the set of all $n \times m$ real matrices. The notation $P>0$ (or $P<0$ ), for any symmetric matrix $P \in R^{n \times n}$, means that $P$ is positive (or negative) definite. The notation $\left(\begin{array}{ll}A & B \\ * & C\end{array}\right)$, for any square matrices $A$ and $B$ in $R^{n \times m}$, stands for $\left(\begin{array}{cc}A & B \\ B^{T} & C\end{array}\right)$. Moreover, the notation $I$ stands for the identity matrix. The expression $e_{i}$ represents $(\underbrace{0, \ldots, 0}_{i-1}, I, \underbrace{0, \ldots, 0}_{n-i})$.

\section{Preliminaries}

In this section, to show the priority of our approach, we consider the following linear time-delay systems:

$$
\begin{aligned}
& \dot{x}(t)=A x(t)+A_{d} x(t-h), \quad \forall t \geq 0, \\
& x(t)=\phi(t), \quad \forall t \in[-h, 0],
\end{aligned}
$$

where $x(t) \in R^{n}$ is the state vector, $\phi$ is the initial condition, and $A, A_{d} \in R^{n \times n}$ are constant matrices. The delay $h$ is a scalar and satisfies the constraint: $h \geq 0$. Before presenting our results, the following lemmas are provided, which are very important to derive the later delay-dependent stability conditions.

Lemma 1 (see [12]). For given symmetric positive definite matrices $R>0$ and any differentiable function $w$ in $[t-h, t] \rightarrow$ $R^{n}$, the following inequality holds:

$$
\begin{aligned}
& -\int_{t-h}^{t} w^{T}(s) R w(s) d s \\
& \quad \leq-\frac{1}{h}\left(\int_{t-h}^{t} w(s) d s\right)^{T} R\left(\int_{t-h}^{t} w(s) d s\right) .
\end{aligned}
$$

Lemma 2 (see [1]). For given symmetric positive definite matrices $R>0$ and any differentiable function $w$ in $[t-h, t] \rightarrow$ $R^{n}$, the following inequality holds:

$$
\int_{t-h}^{t} \int_{\theta}^{t} \dot{w}^{T}(s) R \dot{w}(s) d s d \theta \geq \frac{2}{h^{2}} \Omega_{0}^{T} R \Omega_{0}
$$

where

$$
\Omega_{0}=h w(t)-\int_{t-h}^{t} w(s) d s .
$$

Lemma 3. For given symmetric positive definite matrices $R>$ 0 and any differentiable function $w$ in $[a, b] \rightarrow R^{n}$, the following inequality holds:

$$
\int_{a}^{b} \dot{w}(u)^{T} R \dot{w}(u) d u \geq \frac{3}{4(b-a)} \Omega^{T} K \Omega,
$$

where

$$
\begin{aligned}
\Omega & =\left(w^{T}(b), w^{T}(a), \frac{1}{(b-a)} \int_{a}^{b} w^{T}(u) d u\right)^{T}, \\
K & =\left(\begin{array}{ccc}
5 R & 2 R & -7 R \\
* & 4 R & -6 R \\
* & * & 13 R
\end{array}\right) .
\end{aligned}
$$

Proof. For any differentiable function $w(u) \in W[a, b]$, consider a signal $z(u)$ given by

$$
\begin{aligned}
z(u)= & \dot{w}(u)-\frac{1}{(b-a)} \Omega_{1}+\frac{3 g(u)}{(b-a)^{2}} \Omega_{2} \\
& -\frac{f(u)}{2(b-a)^{2}} \Omega_{3},
\end{aligned}
$$

where

$$
\begin{aligned}
\Omega_{1} & =w(b)-w(a), \\
\Omega_{2} & =w(b)+w(a)-\frac{2}{b-a} \int_{a}^{b} w(u) d u, \\
\Omega_{3} & =2 w(b)+w(a)-\frac{3}{b-a} \int_{a}^{b} w(u) d u, \\
g(u) & =b+a-2 u, \\
f(u) & =3 u-2 a-b .
\end{aligned}
$$

Simple calculus ensures that

$$
\begin{aligned}
\int_{a}^{b} g(u) d u & =0, \\
\int_{a}^{b} g(u)^{2} d u & =\frac{1}{3}(b-a)^{3}, \\
\int_{a}^{b} g(u) \dot{w}(u) d u & =-(b-a) \Omega_{2}, \\
\int_{a}^{b} \dot{w}(u) d u & =w(b)-w(a)=\Omega_{1}, \\
\int_{a}^{b} f(u) d u & =\frac{1}{2}(b-a)^{2}, \\
\int_{a}^{b} f(u)^{2} d u & =(b-a)^{3}, \\
\int_{a}^{b} f(u) \dot{w}(u) d u & =(b-a) \Omega_{3}, \\
\int_{a}^{b} f(u) g(u) d u & =-\frac{1}{2}(b-a)^{3} .
\end{aligned}
$$




$$
\begin{aligned}
& \text { The computation of } \int_{a}^{b} \int_{\theta}^{t} z^{T}(u) R z(u) d u d \theta \text { leads to } \\
& \int_{a}^{b} \int_{\theta}^{t} z^{T}(u) R z(u) d u d \theta \\
& =\int_{a}^{b} \dot{w}(u)^{T} R \dot{w}(u) d u-\frac{1}{b-a} \Omega_{1}^{T} R \Omega_{1} \\
& -\frac{3}{b-a} \Omega_{2}^{T} R \Omega_{2}-\frac{1}{2(b-a)} \Omega_{3}^{T} R \Omega_{3} \\
& -\frac{1}{4(b-a)} \Omega_{1}^{T} R \Omega_{5}-\frac{1}{4(b-a)} \Omega_{3}^{T} R \Omega_{1} \\
& -\frac{3}{4(b-a)} \Omega_{2}^{T} R \Omega_{3}-\frac{3}{4(b-a)} \Omega_{3}^{T} R \Omega_{2} \\
& =\int_{a}^{b} \dot{w}(u)^{T} R \dot{w}(u) d u \\
& -\frac{1}{4(b-a)}\left(\begin{array}{c}
\Omega_{1} \\
\Omega_{2} \\
\Omega_{3}
\end{array}\right)^{T}\left(\begin{array}{ccc}
4 R & 0 & -R \\
* & 12 R & -3 R \\
* & * & 3 R
\end{array}\right)\left(\begin{array}{c}
\Omega_{1} \\
\Omega_{2} \\
\Omega_{3}
\end{array}\right) .
\end{aligned}
$$

Consider now the right-hand side of expression (10); let

$$
\Omega^{T}=\left(w^{T}(b), w^{T}(a), \frac{1}{(b-a)} \int_{a}^{b} w^{T}(u) d u\right),
$$

and the following formulation will be obtained:

$$
\left(\begin{array}{l}
\Omega_{1} \\
\Omega_{2} \\
\Omega_{3}
\end{array}\right)=\left(\begin{array}{ccc}
I & -I & 0 \\
I & I & -2 I \\
2 I & I & -3 I
\end{array}\right) \Omega .
$$

It can be seen that the left-hand side of expression (10) is positive definite since $R>0$. Combining expression (10) and (12), we can complete the proof.

Remark 4. It is worth mentioning that the choice of function $z(u)$ is very necessary to Lemma 3 . On one hand, functions $g(u)$ and $f(u)$ should be constructed so as the cross term $\int_{a}^{b} g(u) d u \Omega_{1}^{T} R \Omega_{2}=0$ and $\int_{a}^{b} f(u) d u \Omega_{2}^{T} R \Omega_{3} \neq 0$ in computing $\int_{a}^{b} z^{T}(u) R z(u) d u$. On the other hand, to unify the coefficient $1 /(b-a)$ of the expression, $z(u)$ should contain the coefficient $1 /(b-a)^{2}$.

Remark 5. Meanwhile, the choice of functions $g(u)$ and $f(u)$ is very important and the coefficient of parameters $u, a$, and $b$ in functions $g(u)$ and $f(u)$ must be ensured so that the sum of the three coefficients is zero. Otherwise, we cannot guarantee that expression (9) is true.

Remark 6. It is interesting that inequality (5) will transform into inequality (2) when $\Omega_{2}=0$ and $\Omega_{3}=0$; this mean that inequality (5) encompass Jensen's inequality. Thus, we can say that inequality (5) improved Jensen's inequality.

\section{Main Results}

This section will state the stability analysis for time-delay systems. In the following context, we aim at assessing the stability of system (1) and illustrating the application and analysis of new integral inequalities combined with a simple LyapunovKrasovskii functional which is constructed by utilizing delaydecomposition approach with uncertain parameter. Based on the previous inequalities, the following theorem is provided.

Theorem 7. For a given constant delay $h$, system (1) is asymptotically stable if there exist $n \times n$-matrices $P_{11}=P_{11}^{T}>0$, $P_{i j}=P_{j i}^{T}, Q_{i}=Q_{i}^{T}>0,(i, j=1,2)$, and $S=S^{T}>0$ such that the following conditions hold:

$\Phi$

$$
=\left(\begin{array}{ccc}
P_{11}+2 S & P_{12}-\frac{2}{h} S & P_{12}-\frac{2}{h} S \\
* & P_{22}+\frac{1}{\alpha h} Q_{1}+\frac{2}{h^{2}} S & P_{22}+\frac{2}{h^{2}} S \\
* & * & P_{22}+\frac{1}{(1-\alpha) h} Q_{2}+\frac{2}{h^{2}} S
\end{array}\right)
$$

$>0$,

$$
\Psi=\left(\begin{array}{ccccc}
M_{11} & M_{12} & M_{13} & M_{14} & M_{15} \\
* & M_{22} & M_{23} & M_{24} & M_{25} \\
* & * & M_{33} & M_{34} & M_{35} \\
* & * & * & M_{44} & 0 \\
* & * & * & * & M_{55}
\end{array}\right)<0
$$

where

$$
\begin{aligned}
M_{11}= & A^{T} P_{11}+P_{11} A+P_{12}^{T}+P_{12}+Q_{1}+h A^{T} S A \\
& -\frac{15}{4 \alpha h} S, \\
M_{12}= & -\frac{3}{2 \alpha h} S, \\
M_{22}= & -Q_{1}+Q_{2}-\frac{3}{\alpha h} S-\frac{15}{4(1-\alpha) h} S, \\
M_{13}= & P_{11} B-P_{12}+h A^{T} S B, \\
M_{23}= & -\frac{3}{2(1-\alpha) h} S, \\
M_{33}= & -Q_{2}+h B^{T} S B-\frac{3}{(1-\alpha) h} S, \\
M_{14}= & \alpha h\left(A^{T} P_{12}+P_{22}\right)+\frac{21}{4 \alpha h} S, \\
M_{24}= & \frac{9}{2 \alpha h} S, \\
M_{34}= & \alpha h\left(B^{T} P_{12}-P_{22}\right), \\
M_{44}= & -\frac{39}{4 \alpha h} S,
\end{aligned}
$$




$$
\begin{aligned}
& M_{15}=(1-\alpha) h\left(A^{T} P_{12}+P_{22}\right), \\
& M_{25}=\frac{21}{4(1-\alpha) h} S, \\
& M_{35}=(1-\alpha) h\left(B^{T} P_{12}-P_{22}\right)+\frac{9}{2(1-\alpha) h} S, \\
& M_{55}=-\frac{39}{4(1-\alpha) h} S .
\end{aligned}
$$

Proof. Consider the following Lyapunov-Krasovskii functional given by

$$
V\left(t, x_{t}\right)=V_{1}\left(t, x_{t}\right)+V_{2}\left(t, x_{t}\right)+V_{3}\left(t, x_{t}\right)
$$

where

$$
\begin{aligned}
& V_{1}\left(t, x_{t}\right)=\left(\begin{array}{c}
x(t) \\
\int_{t-h}^{t} x(s) d s
\end{array}\right)^{T} \\
& \cdot\left(\begin{array}{cc}
P_{11} & P_{12} \\
* & P_{22}
\end{array}\right)\left(\int_{t-\alpha h}^{t} x(s) d s+\int_{t-h}^{t-\alpha h} x(s) d s\right), \\
& V_{2}\left(t, x_{t}\right)=\int_{t-\alpha h}^{t} x^{T}(s) Q_{1} x(s) d s \\
& +\int_{t-h}^{t-\alpha h} x^{T}(s) Q_{2} x(s) d s, \\
& V_{3}\left(t, x_{t}\right)=\int_{t-h}^{t} \int_{\theta}^{t} \dot{x}^{T}(s) S \dot{x}(s) d s d \theta .
\end{aligned}
$$

Let

$$
\zeta^{T}=\left(x^{T}(t), \int_{t-\alpha h}^{t} x^{T}(s) d s, \int_{t-h}^{t-\alpha h} x^{T}(s) d s\right)
$$

and then employing Lemmas 1 and 2, respectively, to the above expression, we can obtain

$$
\begin{aligned}
& V_{1}\left(t, x_{t}\right)=\zeta^{T}\left(\begin{array}{c}
e_{1} \\
e_{2}+e_{3}
\end{array}\right)^{T}\left(\begin{array}{cc}
P_{11} & P_{12} \\
* & P_{22}
\end{array}\right)\left(\begin{array}{c}
e_{1} \\
e_{2}+e_{3}
\end{array}\right) \zeta, \\
& V_{2}\left(t, \dot{x}_{t}\right) \geq \frac{1}{\alpha h}\left(\int_{t-\alpha h}^{t} x(s) d s\right)^{T} Q_{1}\left(\int_{t-\alpha h}^{t} x(s) d s\right) \\
& +\frac{1}{(1-\alpha) h}\left(\int_{t-h}^{t-\alpha h} x(s) d s\right)^{T} Q_{2}\left(\int_{t-h}^{t-\alpha h} x(s) d s\right) \\
& \quad=\zeta^{T}\left[\frac{1}{\alpha h} e_{2}^{T} Q_{1} e_{2}+\frac{1}{(1-\alpha) h} e_{3}^{T} Q_{2} e_{3}\right] \zeta, \\
& V_{3}\left(t, \dot{x}_{t}\right) \geq \frac{2}{h^{2}} \Omega_{4}^{T} S \Omega_{4},
\end{aligned}
$$

where

$$
\begin{aligned}
\Omega_{4} & =h x(t)-\int_{t-h}^{t} x(s) d s \\
& =h x(t)-\int_{t-\alpha h}^{t} x(s) d s-\int_{t-h}^{t-\alpha h} x(s) d s \\
& =\left[h e_{1}-e_{2}-e_{3}\right] \zeta .
\end{aligned}
$$

By (18), we obtain the following lower bound of $V\left(t, x_{t}\right)$ : $V\left(t, x_{t}\right) \geq \zeta^{T} \Phi \zeta$. It is easy to see that the positive definiteness of the matrices $\Phi$ implies the positive definiteness of the functional $V\left(t, x_{t}\right)$.

Let

$$
\begin{aligned}
\xi^{T} & =\left(x^{T}(t), x^{T}(t-\alpha h), x^{T}(t-h), \frac{1}{\alpha h}\right. \\
& \left.\cdot \int_{t-\alpha h}^{t} x^{T}(s) d s, \frac{1}{(1-\alpha) h} \int_{t-h}^{t-\alpha h} x^{T}(s) d s\right) .
\end{aligned}
$$

Calculating the derivative of the functional along the trajectories of system (1) and applying Lemma 3,

$$
\begin{aligned}
& \dot{V}_{1}\left(t, x_{t}\right)=2\left(\begin{array}{c}
\dot{x}(t) \\
x(t)-x(t-h)
\end{array}\right)^{T} \\
& \cdot P\left(\int_{t-\alpha h}^{t} x(s) d s+\int_{t-h}^{t-\alpha h} x(s) d s\right) \\
& =2 \xi^{T}\left[\left(\begin{array}{c}
A e_{1}+B e_{3} \\
e_{1}-e_{3}
\end{array}\right)^{T} P\left(\begin{array}{c}
e_{1} \\
\alpha h e_{4}+(1-\alpha) h e_{5}
\end{array}\right)\right] \xi, \\
& \dot{V}_{2}\left(t, x_{t}\right)=x^{T}(t) Q_{1} x(t)-x^{T}(t-\alpha h)\left(Q_{1}-Q_{2}\right) x(t \\
& -\alpha h)-x^{T}(t-h) Q_{2} x(t-h)=\xi^{T}\left[e_{1}^{T} Q_{1} e_{1}\right. \\
& \left.-e_{2}^{T}\left(Q_{1}-Q_{2}\right) e_{2}-e_{3}^{T} Q_{2} e_{3}\right] \xi \\
& \dot{V}_{3}\left(t, x_{t}\right)=h \dot{x}^{T}(t) S \dot{x}(t)-\int_{t-\alpha h}^{t} \dot{x}^{T}(s) S \dot{x}(s) d s \\
& -\int_{t-h}^{t-\alpha h} \dot{x}^{T}(s) S \dot{x}(s) d s \leq h(A x(t) \\
& +B x(t-h))^{T} S(A x(t)+B x(t-h))-\frac{1}{\alpha h} \Omega_{5}^{T} K \Omega_{5} \\
& -\frac{1}{(1-\alpha) h} \Omega_{6}^{T} K \Omega_{6} \\
& =\xi^{T}\left[h\left(A e_{1}+B e_{3}\right)^{T} S\left(A e_{1}+B e_{3}\right)-\frac{1}{\alpha h} \Omega_{1}^{T} K \Omega_{1}\right. \\
& \left.-\frac{1}{(1-\alpha) h} \Omega_{2}^{T} K \Omega_{2}\right] \xi \text {, }
\end{aligned}
$$


where

$$
\begin{aligned}
\Omega_{5} & =\left(x^{T}(t), x^{T}(t-\alpha h), \frac{1}{\alpha h} \int_{t-\alpha h}^{t} x^{T}(s) d s\right) \\
& =\left(e_{1}, e_{2}, e_{4}\right) \xi \\
\Omega_{6} & =\left(x^{T}(t-\alpha h), x^{T}(t-h), \frac{1}{(1-\alpha) h}\right. \\
& \left.\cdot \int_{t-h}^{t-\alpha h} x^{T}(s) d s\right)=\left(e_{2}, e_{3}, e_{5}\right) \xi \\
P & =\left(\begin{array}{cc}
P_{11} & P_{12} \\
* & P_{22}
\end{array}\right), \\
K_{1} & =\frac{3}{4}\left(\begin{array}{rrr}
5 S & 2 S & -7 S \\
* & 4 S & -6 S \\
* & * & 13 S
\end{array}\right) .
\end{aligned}
$$

Combined with (21), we can get $\dot{V}\left(t, x_{t}\right) \leq \xi^{T} \Psi \xi$. Therefore, system (1) is asymptotic from the Lyapunov-Krasovskii stability theorem [12] if $\Psi<0$ holds. This completes the proof.

Remark 8. It is worth noting that the construction of our Lyapunov-Krasovskii functionals is very general and simple, especially the functionals $V_{1}\left(t, x_{t}\right)$ and $V_{2}\left(t, x_{t}\right)$, which play an important role in reducing the conservativeness of stability criterion for neutral time-delay systems, since the functional includes more cross quadratic terms.

Remark 9. To illustrate the effectiveness and the less conservativeness of our new inequality, constructing the functional $V_{3}\left(t, x_{t}\right)$ is important and necessary, because it can induce single integral term, and then we can use the delaydecomposition approach and our new inequality.

Remark 10. The delay-decomposition approach with uncertain parameter has been applied to construct the LyapunovKrasovskii functionals such that the discussing interval of the delay of time-delay systems is extended to two segments, and according to different parameter value we can get more accurate and better results of delay $h$.

\section{Example}

In this section, our main purpose is to show how the inequality is given in Section 2 and the delay-decomposition approach with uncertain parameter reduces the conservatism in the stability condition. Furthermore, the simulated picture is provided to visually illustrate the effectiveness of our results.

Example 1. Consider the following example of the time-delay systems (1) with the matrices

$$
\begin{aligned}
& A=\left(\begin{array}{cc}
-2 & 0 \\
0 & -0.9
\end{array}\right), \\
& B=\left(\begin{array}{cc}
-1 & 0 \\
-1 & -1
\end{array}\right) .
\end{aligned}
$$

TABLE 1: The maximal allowed delays of example (23) for different values of delay $h$.

\begin{tabular}{lcc}
\hline Theorems & $h_{\max }$ & Number of variables \\
\hline$[19]$ & 6.11 & $1.5 n^{2}+9 n+9$ \\
{$[21] D_{d}=3$} & 5.96 & $6.5 n^{2}+2.5 n$ \\
{$[21] D_{d}=4$} & 6.05 & $10 n^{2}+3 n$ \\
{$[24] D_{d}=1$} & 6.053 & $7.5 n^{2}+3.5 n$ \\
{$[24] D_{d}=2$} & 6.165 & $10.5 n^{2}+4.5 n$ \\
{$[24] D_{d}=3$} & 6.171 & $14.5 n^{2}+4.5 n$ \\
{$[24] D_{d}=4$} & 6.171 & $20.5 n^{2}+5.5 n$ \\
{$[15] D_{p}=2$} & 5.90 & $12.5 n^{2}+4.5 n$ \\
{$[15] D_{p}=3$} & 6.10 & $21 n^{2}+6 n$ \\
{$[32](N=1)$} & 6.059 & $3 n^{2}+2 n$ \\
{$[32](N=2)$} & 6.168 & $5.5 n^{2}+2.5 n$ \\
\hline Theorem $7(\alpha=0.6563)$ & 6.1721 & $3 n^{2}+3 n$ \\
\hline Theorem $7(\alpha=0.6564)$ & 6.1719 & $3 n^{2}+3 n$ \\
\hline Theorem $7(\alpha=0.6565)$ & 6.1716 & $3 n^{2}+3 n$ \\
\hline
\end{tabular}

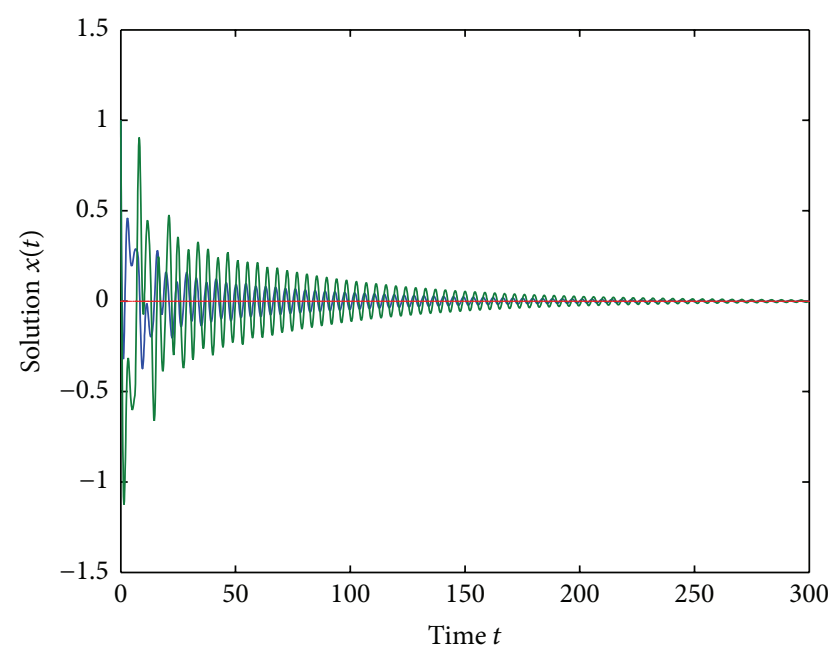

FIGURE 1: Example (23) for delay $h=h_{\max }=6.1721$.

This system is a well-known delay-dependent stable timedelay system. That is, the delay free system is stable and the maximum allowable delay $h_{\max }=6.1721$ can be easily computed by delay sweeping techniques. To intuitively demonstrate the effectiveness and veracity of our approach, we will compare the results with the literature in Table 1 and the related simulated picture is reported in Figure 1.

In Table 1 , the notation $D_{p}$ in [15] means that the degree of freedom comes from the degree of the polynomial matrices, and the notation $D_{d}$ in $[21,24]$ means that the degree of the polynomial is always 1 , but the degree of freedom comes from the degree of discretization. Apparently, Table 1 shows that our result is competitive with the most accurate stability conditions from the literature. For the case of constant and known delay, it delivers a better result of delay $h$ than the one provided by other theorems, such as $[15,19,21,24,32]$, and it has a lower number of variables although the result of $h$ in our paper is a little smaller than the other results. 
Meanwhile, it is interesting that the simulated figure describes a distinct phenomenon that the system tends to be stable within a limited time; please look at Figure 1. In other words, our result is very suitable for time-delay systems producing some less conservative conditions than those from these literatures.

\section{Conclusion}

In this paper, we provide a new useful integral inequality, which has been proved to be suitable for the stability analysis of time-delay systems. Then, we construct a simple LyapunovKrasovskii functional and derive new stability criteria for time-delay systems, by employing this new integral inequality and combining with delay-decomposition approach with uncertain parameter. The new result we gained has been expressed in terms of LMIs and shows a larger improvement than the existing results through a numerical example. More generally, this new inequality could be coupled to more elaborated Lyapunov functional to resolve more problems about time-delay systems.

\section{Conflict of Interests}

The authors declare that there is no conflict of interests regarding the publication of this paper.

\section{Acknowledgments}

This research is supported by the Natural Science Foundation of Yunnan Province (Grant no. 2011FZ172); the Specialized Research Fund For the Doctoral Program of Higher Education (Grant no. 135578); the National Natural Science Fund of China (Grant no. 11461082); the National Natural Science Fund of China (Grant no. 11461083); the Scientific Research Fund Project in Yunnan Provincial Department of Education (Grant no. 2015J069).

\section{References}

[1] B. Ni and Q. Han, "On stability for a class of neutral delaydifferential systems," in Proceedings of the American Control Conference, vol. 6, pp. 4544-4549, Arlington, Va, USA, June 2001.

[2] E. Fridman and U. Shaked, "An improved stabilization method for linear time-delay systems," IEEE Transactions on Automatic Control, vol. 47, no. 11, pp. 1931-1937, 2002.

[3] A. H. Glattfelder and W. Schaufelberger, "Stability analysis of single loop control systems with saturation and antiresetwindup circuits," IEEE Transactions on Automatic Control, vol. 28, no. 12, pp. 1074-1081, 1983.

[4] J. G. da Silva Jr., E. Fridman, A. Seuret, and J. Richard, "Stabilization of neutral systems with saturating inputs," in Proceedings of the 16th IFAC World Congress (IFAC'05), Prague, Czech Republic, June 2005.

[5] K. Gu and S.-I. Niculescu, "Additional dynamics in transformed time-delay systems," IEEE Transactions on Automatic Control, vol. 45 , no. 3, pp. $572-575,2000$.
[6] B. Goubet, M. Dambrin, and J. Richard, "Stability of perturbed systems with time-varying delays," Systems and Control Letters, vol. 31, pp. 155-163, 1997.

[7] Q.-L. Han, "Robust stability of uncertain delay-differential systems of neutral type," Automatica, vol. 38, no. 4, pp. 719-723, 2002.

[8] A. Haurani, H. H. Michalska, and B. Boulet, "Delay-dependent robust stabilization of uncertain neutral systems with saturating actuators," in Proceedings of the American Control Conference (ACC '03), vol. 1, pp. 509-514, IEEE, Denver, Colo, USA, June 2003.

[9] N. J. Krikelis, "State feedback integral control with intelligent integrator," International Journal of Control, vol. 32, no. 3, pp. 465-473, 1980.

[10] P.-L. Liu, "Stabilization of input delay constrained systems with delay dependence," International Journal of Systems Science, vol. 26, no. 2, pp. 245-255, 1995.

[11] C.-H. Lien, K.-W. Yu, and J.-G. Hsieh, "Stability conditions for a class of neutral systems with multiple time delays," Journal of Mathematical Analysis and Applications, vol. 245, no. 1, pp. 2027, 2000.

[12] K. Gu, J. Chen, and V. Kharitonov, Stability of Time-Delay Systems, Birkhäuser, Boston, Mass, USA, 2003.

[13] T. Su, C. Lu, and J. Tsai, "Delay-dependent robust criteria for uncertain systems with multiple state delays," in Proceedings of the American Control Conference (ACC '01), vol. 1, pp. 25-27, Arlington, Va, USA, June 2001.

[14] S. Tarbouriech and G. Garcia, "Stabilization of neutral linear time-delay systems with saturating actuators," in Proceedings of the 38th IEEE Conference on Decision and Control (CDC '99), pp. 2011-2016, Phoenix, Ariz, USA, December 1999.

[15] M. M. Peet, A. Papachristodoulou, and S. Lall, "Positive forms and stability of linear time-delay systems," SIAM Journal on Control and Optimization, vol. 47, no. 6, pp. 3237-3258, 2009.

[16] J.-J. Yan, "Robust stability analysis of uncertain time delay systems with delay-dependence," Electronics Letters, vol. 37, no. 2, pp. 135-137, 2001.

[17] J.-J. Yan, J.-S. Lin, and T.-L. Liao, "Robust dynamic compensator for a class of time delay systems containing saturating control input," Chaos, Solitons \& Fractals, vol. 31, no. 5, pp. 1223-1231, 2007.

[18] J. Sun, G. P. Liu, J. Chen, and D. Rees, "Improved delay-rangedependent stability criteria for linear systems with time-varying delays," Automatica, vol. 46, no. 2, pp. 466-470, 2010.

[19] C.-Y. Kao and A. Rantzer, "Stability analysis of systems with uncertain time-varying delays," Automatica, vol. 43, no. 6, pp. 959-970, 2007.

[20] F. Gouaisbaut and D. Peaucelle, "A note on stability of time delay systems," in Proceedings of the 5th IFAC Symposium on Robust Control Design (ROCOND '06), pp. 555-560, Toulouse, France, 2006.

[21] Q.-L. Han, "A discrete delay decomposition approach to stability of linear retarded and neutral systems," Automatica, vol. 45, no. 2, pp. 517-524, 2009.

[22] J. Sun, G.-P. Liu, and J. Chen, "Delay-dependent stability and stabilization of neutral time-delay systems," International Journal of Robust and Nonlinear Control, vol.19, no. 12, pp.1364$1375,2009$.

[23] K. Gu, "An integral inequality in the stability problem of timedelay systems," in Proceedings of the 39th IEEE Conference on Decision and Control, vol. 3, pp. 2805-2810, IEEE, Sydney, Australia, December 2000. 
[24] K. Gu, "A further refinement of discretized lyapunov functional method for the stability of time-delay systems," International Journal of Control, vol. 74, no. 10, pp. 967-976, 2001.

[25] M. Wu, Y. He, J.-H. She, and G.-P. Liu, "Delay-dependent criteria for robust stability of time-varying delay systems," Automatica, vol. 40, no. 8, pp. 1435-1439, 2004.

[26] Y. He, Q.-G. Wang, C. Lin, and M. Wu, "Delay-range-dependent stability for systems with time-varying delay," Automatica, vol. 43, no. 2, pp. 371-376, 2007.

[27] Y. He, Q.-G. Wang, L. Xie, and C. Lin, "Further improvement of free-weighting matrices technique for systems with timevarying delay," IEEE Transactions on Automatic Control, vol. 52, no. 2, pp. 293-299, 2007.

[28] J. C. J. Sun, G. P. Liu, J. Chen, and D. Rees, "Improved delayrange-dependent stability criteria for linear systems with timevarying delays," Automatica, vol. 46, no. 2, pp. 466-470, 2010.

[29] H. Fujioka, "Stability analysis of systems with aperiodic sampleand-hold devices," Automatica, vol. 45, no. 3, pp. 771-775, 2009.

[30] Y. Ariba and F. Gouaisbaut, "An augmented model for robust stability analysis of time-varying delay systems," International Journal of Control, vol. 82, no. 9, pp. 1616-1626, 2009.

[31] Y. Ariba and F. Gouaisbaut, "Input-output framework for robust stability of time-varying delay systems," in Proceedings of the 48th IEEE Conference on Decision and Control (CDC '09), pp. 274-279, Shanghai, China, December 2009.

[32] A. Seuret and F. Gouaisbaut, "Hierarchy of LMI conditions for the stability analysis of time-delay systems," Systems \& Control Letters, vol. 81, pp. 1-7, 2015. 


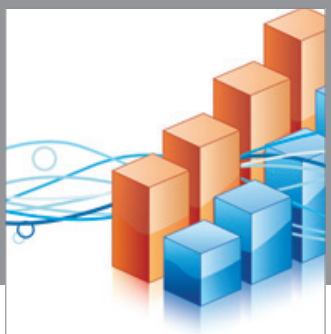

Advances in

Operations Research

vatem alat4

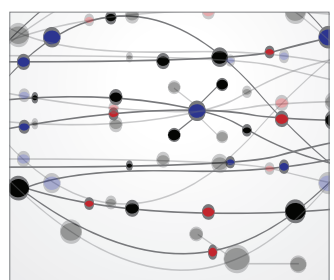

\section{The Scientific} World Journal
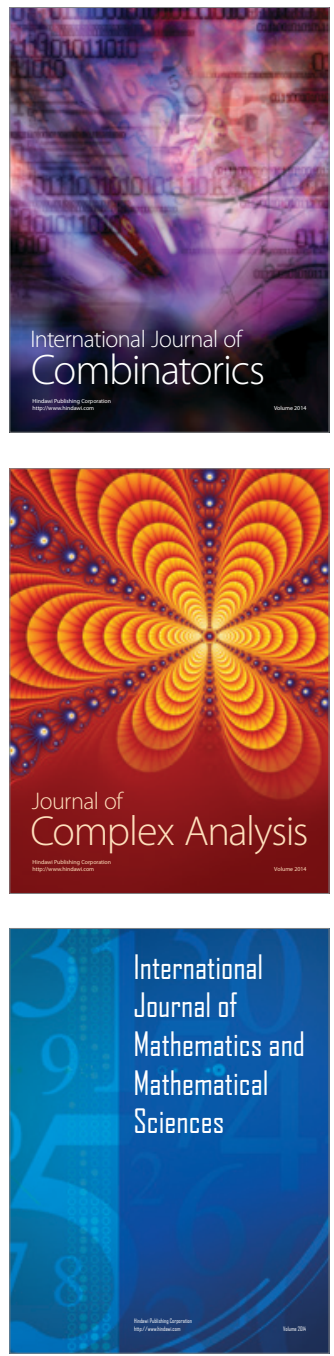
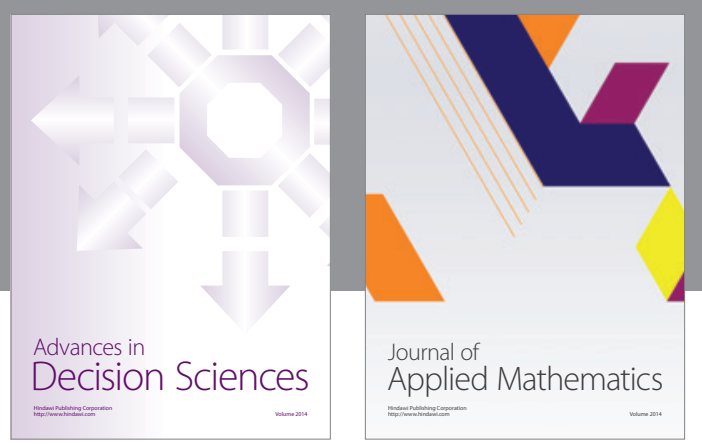

Algebra

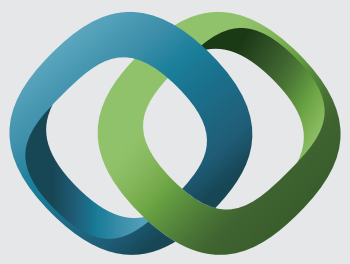

\section{Hindawi}

Submit your manuscripts at

http://www.hindawi.com
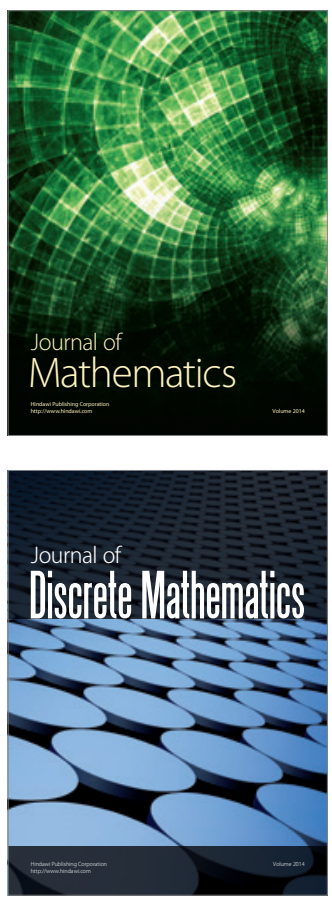

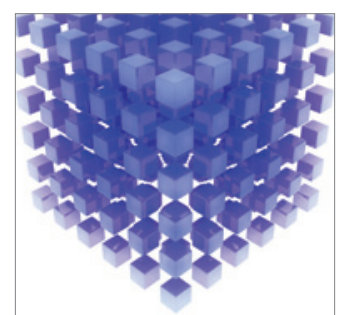

Mathematical Problems in Engineering
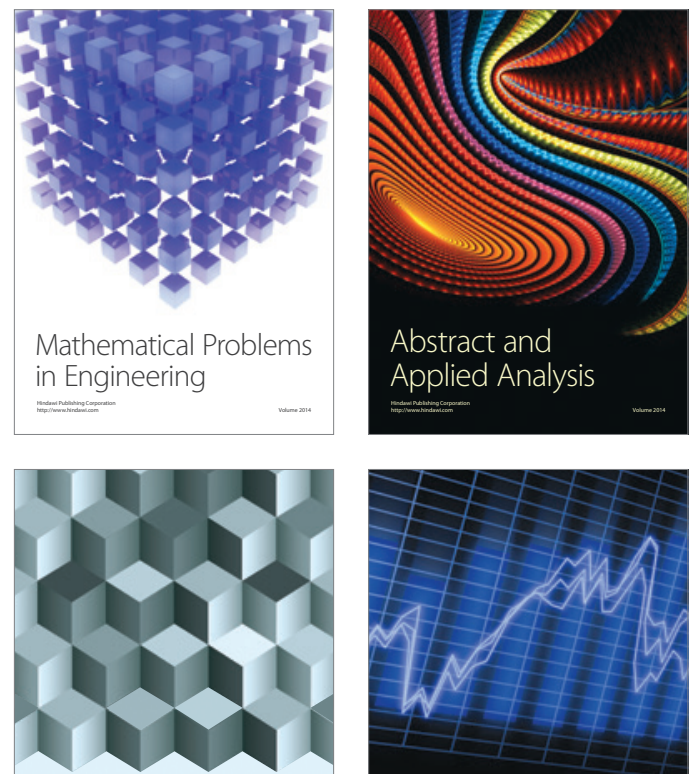

Journal of

Function Spaces

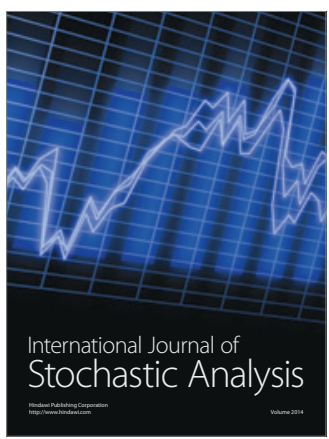

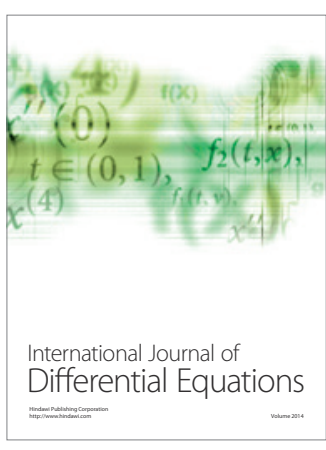
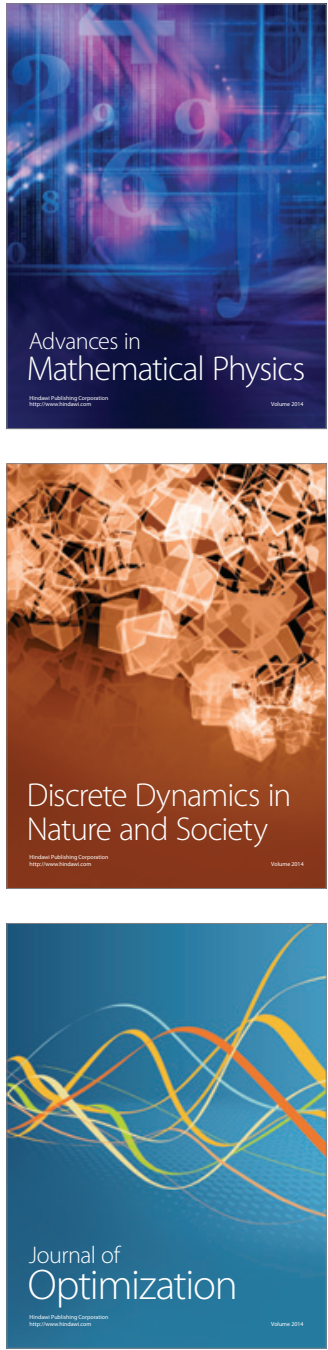